\title{
Multidrug-Resistant Orthopaedic Surgical Site Infections Treated with Linezolid in Four Dogs
}

\author{
Po-Yen Chou ${ }^{1}$ Jin Yoon ${ }^{1, *(1)}$ Rebecca A. Hersh-Boyle ${ }^{1, *}$ Denis J. Marcellin-Little ${ }^{1}$ (1) \\ ${ }^{1}$ Department of Veterinary Surgical and Radiological Science, School \\ of Veterinary Medicine, University of California, Davis, California, \\ United States \\ Address for correspondence Po-Yen Chou, BVM, MS, DACVS-SA, \\ Department of Veterinary Surgical and Radiological Science, School of \\ Veterinary Medicine, University of California, Davis, CA 95616-5270, \\ United States (e-mail: pchou@ucdavis.edu).
}

VCOT Open 2020;3:e72-e76.

\begin{abstract}
Keywords

- infections

- multiresistant bacteria

- surgical site infection

- dog

- linezolid

Objective This study aimed to report the adverse drug events and treatment outcome of systemic linezolid therapy to manage multi-drug resistant orthopaedic surgical site infection in dogs.

Materials and Methods Retrospective case review of four dogs that received linezolid to treat surgical site infections after orthopaedic surgery. Reevaluations consisted of a clinical examination or a telephone interview.

Results Serum drug concentrations varied. All dogs showed a temporary resolution of clinical signs of surgical site infection. Two dogs that received linezolid at the previously reported dose developed drug-associated side effects. The side effects were anorexia, nausea, vomiting and regenerative anaemia. All side effects resolved after the discontinuation of linezolid. Surgical site infection recurred in two dogs 52 and 177 days after discontinuing linezolid respectively.

Clinical significance Adverse drug events occurred in dogs receiving oral linezolid at the dosage of 10 to $20 \mathrm{mg} / \mathrm{kg}$. Oral linezolid therapy failed to resolve deep orthopaedic surgical site infections in two out of four dogs. As a tertiary antimicrobial, linezolid should only be used in carefully selected cases while monitoring for drug-associated side effects.
\end{abstract}

\section{Introduction}

Linezolid is a synthetic oxazolidinone antimicrobial agent that inhibits protein synthesis by binding to ribosomal 50S subunits, which leads to inhibition of 70S subunit initiation and messenger ribonucleic acid translation. ${ }^{1}$ Linezolid is an effective antimicrobial against multidrug-resistant Gram-positive bacteria, including methicillin-resistant Staphylococcus (MRS) and vancomycin-resistant Enterococcus species. ${ }^{2}$ The use of linezolid has been reported in dogs for the treatment of MRS infections such as discospondylitis and deep pyoderma. The

J.Y.'s current workplace is VCA West Coast Specialty and Emergency Animal Hospital, Orange County, California, United States. R.A.H-B's current workplace is the Sam's Clinic, Marin, California, United States.

received

February 19, 2020

accepted after revision

June 8,2020 reported oral linezolid dosage in dogs ranged from 10 to $20 \mathrm{mg} / \mathrm{kg}$ every 8 to 12 hours. ${ }^{3,4}$ In human patients, systemic linezolid therapy can cause side effects such as diarrhoea, vomiting, urticaria, peripheral neuropathy, anaemia, leukopenia and thrombocytopenia. ${ }^{5}$ However, information describing the adverse effects of linezolid in dogs is lacking. ${ }^{4}$ The purpose of this case series is to report the efficacy and adverse effects associated with long-term oral linezolid therapy for the treatment of resistant implant associated orthopaedic related surgical site infections (SSI) in four dogs.

\section{Materials and Methods}

This is a retrospective observational case series. Medical records of dogs that received linezolid for the treatment of SSI following orthopaedic procedures between June 2017

\footnotetext{
(c) 2020 Georg Thieme Verlag KG Stuttgart · New York
}

License terms

10.1055/s-0040-1714141. ISSN 2625-2325.

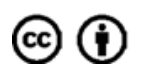


and July 2019 at UC Davis William R. Pritchard Veterinary Medical Teaching Hospital were reviewed. Patient signalment, body weight, orthopaedic procedures preceding SSI, clinical signs and duration of those clinical signs before linezolid administration were retrieved. Bacterial culture and sensitivity, the dosage of oral linezolid administration, trough serum linezolid levels, side effects of linezolid administration, time until remission of clinical signs associated with SSI and duration of linezolid administration were recorded. Follow-up included a physical examination or a telephone interview with the referring veterinarian.

\section{Results}

Four dogs that received linezolid for the treatment of orthopaedic SSI were identified. Signalment, diagnostic findings, antimicrobial and surgical procedures prior to linezolid treatment are summarized in - Table $\mathbf{1}$. All dogs presented with lameness of the affected limb. Two out of four dogs (No. 1 and 2) had surgical implants present following prior stifle surgeries, and septic arthritis was diagnosed based on synovial fluid cytology. One dog (No. 3) presented after an infected cementless total hip replacement. The implants were explanted by the referring veterinarian. The dog continued to have recurrent draining tracts despite daily amikacin injection. One dog (No.4) presented with swelling, redness and drainage after pancarpal arthrodesis plate removal. Empirical antimicrobials administered prior to deep culture included clindamycin in one $\operatorname{dog}$ (No. 1) and amoxicillin/clavulanic acid in another (No. 4). The median number of surgeries performed at the surgical site was three (range: 2-6 surgeries).

Surgery was performed in all dogs to debride unhealthy tissues and collect a deep tissue sample for bacterial culture and sensitivity. Of the two dogs that had orthopaedic implants, implants were removed at the time of surgical debridement in one $\operatorname{dog}$ (No.1) and in the other (No.2), 105 days after the initial debridement surgery due to a delayed union of the osteotomy site. Vancomycin impregnated pluronic gel (Vetrigel, Royer Biomedical, Frederick, Maryland, United States) was implanted locally in two dogs (No.3 and 4) during the debridement surgery. Methicillin-resistant Staphylococcus pseudintermedius (MRSP) was the only bacteria isolated in three dogs (No.1,2 and 4). MRSP, Enterococcus faecalis and Proteus mirabilis were isolated in one dog (No. 3). All cultured MRSP and Enterococcus faecalis were sensitive to linezolid (-Table 2). At least one systemic antimicrobial treatment selected based on bacterial culture and sensitivity reports (Sensititre Companion Animal Gram Positive COMPGP1G Vet AST plate, ThermoFisher Scientific, Waltham, Massachusetts, United States; Sensititre Gram Positive MIC Plate, ThermoFisher Scientific, Waltham, Massachusetts, United States) was administered to all dogs before linezolid therapy (Zyvox, Pfizer, New York, New York, United States). In two dogs, 4 weeks of amikacin were administered intramuscularly (No. 2) and subcutaneously (No. 3). One dog (No. 1) received 4 weeks of trimethoprim-sulfa and subsequently 4 weeks of intravenous amikacin injection. One dog (No. 4) received 4 weeks of trimethoprim-sulfa treatment. The owner declined amikacin injection due to the dog's temperament. The median duration of bacterial infection before linezolid therapy was 206 days (range: 65-659 days).

Linezolid was administered at a dose ranging from 8 to $14 \mathrm{mg} / \mathrm{kg}$ every 12 hours. For dog No. 3, cefpodoxime was also given at $5 \mathrm{mg} / \mathrm{kg}$ every 24 hours to treat Proteus mirabilis. Serum linezolid trough level was measured 2 weeks after initiation of therapy using liquid chromatography (TSQ Quantum Triple Quadrupole Mass Spectrometer, Thermo Finnagan, San Jose, California, United States). In two dogs (No. 1 and 2), serum trough level was measured again 2 weeks after a dose adjustment due to systemic side effects ( - Table 3 ).

All dogs showed improved lameness and resolution of draining tracts after linezolid therapy. The median duration of linezolid treatment was 63 days (range: 42-119 days). Adverse drug events associated with antimicrobial treatment occurred in two dogs (No. 1 and 2). The dogs developed vomiting, lethargy and anorexia 5 to 7 days after linezolid therapy. The side effects were refractory to oral omeprazole and maropitant therapy and resolved once the linezolid dose was decreased from 14 to $7 \mathrm{mg} / \mathrm{kg}$ every 12 hours in one dog (No. 1) and from 13 to $9 \mathrm{mg} / \mathrm{kg}$ every 12 hours in another (No. 2). One dog (No. 1) developed a deep Pseudomonas aeruginosa pyoderma over the tail base and dorsal lumbar region 4 weeks after linezolid therapy. The deep pyoderma resolved after discontinuing underwater treadmill therapy and 6 weeks of enteral marbofloxacin treatment at $5.5 \mathrm{mg} / \mathrm{kg}$ every 24 hours concomitantly with linezolid therapy. One dog (No. 2) developed a macrocytic, hypochromic, regenerative anaemia after

Table 1 Patient background of four dogs receiving linezolid to manage a surgical site infection

\begin{tabular}{|l|l|l|l|l|l|}
\hline Number & Signalment & Surgery & $\begin{array}{l}\text { Number of } \\
\text { surgeries }\end{array}$ & $\begin{array}{l}\text { Duration of } \\
\text { infection (days) }\end{array}$ & Prior antimicrobials \\
\hline 1 & 4-year-old FS Mastiff & TPLO, meniscectomy & 3 & 322 & $\begin{array}{l}\text { Clindamycin } \\
\text { Trimethoprim-sulfa } \\
\text { Amikacin }\end{array}$ \\
\hline 2 & 3-year-old MI Mastiff & $\begin{array}{l}\text { TPLO, extracapsular } \\
\text { stabilization }\end{array}$ & 2 & 65 & Amikacin \\
\hline 3 & 2-year-old FS St. Bernard & Total hip replacement & 6 & 659 & Amikacin \\
\hline 4 & $\begin{array}{l}\text { 5-year-old MI German } \\
\text { Shepherd dog }\end{array}$ & Pancarpal arthrodesis & 2 & 90 & $\begin{array}{l}\text { Amoxicillin/clavulanic acid } \\
\text { Trimethoprim-sulfa }\end{array}$ \\
\hline
\end{tabular}

Abbreviations: FS, female spayed; MI, male intact; TPLO, tibial plateau levelling osteotomy. 


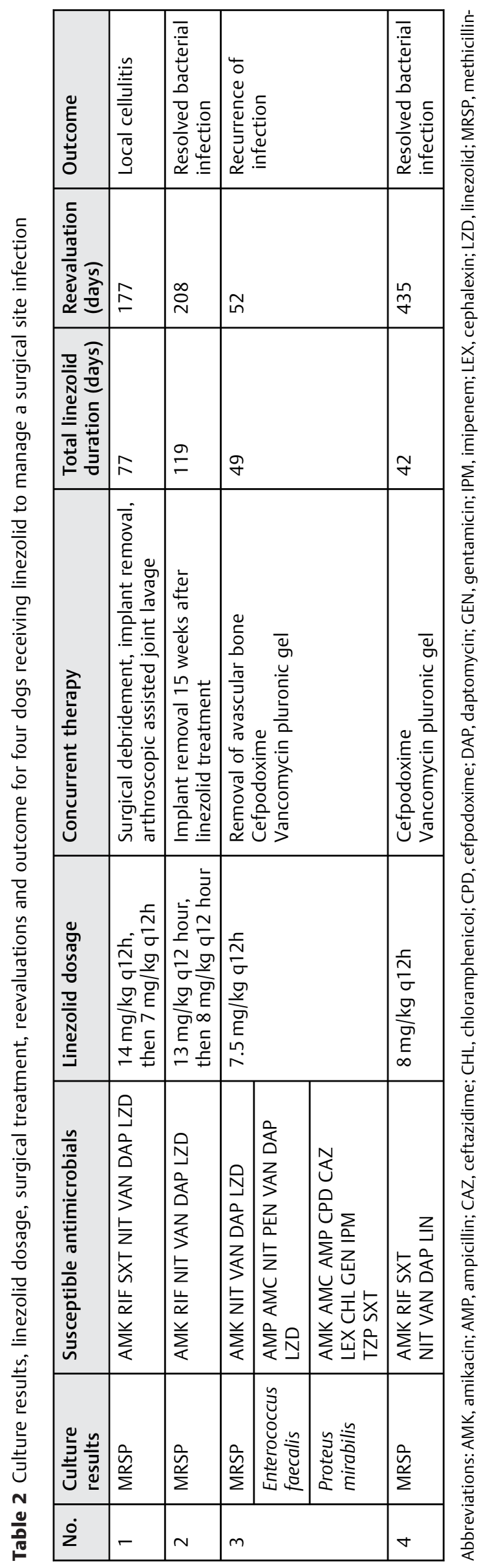

Table 3 In vitro minimal inhibitory concentration against cultured methicillin-resistant Staphylococcus pseudintermedius, dosage and serum trough level during linezolid therapy in four dogs

\begin{tabular}{|l|l|l|l|}
\hline $\begin{array}{l}\text { Dog } \\
\text { number }\end{array}$ & $\begin{array}{l}\text { MIC } \\
(\mu \mathrm{g} / \mathrm{mL})^{\mathrm{a}}\end{array}$ & $\begin{array}{l}\text { Dose } \\
(\mathrm{mg} / \mathrm{kg})\end{array}$ & $\begin{array}{l}\text { Trough level } \\
(\boldsymbol{\mu g} / \mathrm{mL})\end{array}$ \\
\hline \multirow{2}{*}{1} & 1 & 14 & 34.5 \\
\cline { 3 - 4 } & & 7 & 23.7 \\
\hline 2 & 1 & 13 & 16.7 \\
\cline { 3 - 4 } & & 9 & 10.2 \\
\hline \multirow{2}{*}{3} & 1 & 7.5 & 2.86 \\
\cline { 3 - 4 } & & 7.5 & 2.3 \\
\hline 4 & 1 & 8 & 5.2 \\
\hline
\end{tabular}

Abbreviations: MIC, minimum inhibitory concentration. ${ }^{\mathrm{a}} \mathrm{MIC}$ of linezolid reported from surgical sample culture.

105 days of linezolid therapy. The anaemia resolved 14 days after discontinuation of linezolid administration.

The median follow-up after discontinuing all antimicrobial treatment was 127 days (range: 52-435 days). Two dogs (No. 2 and 4) had complete resolution of SSI. Infection recurred in two dogs. In one dog (No. 1), an acute lameness and marked swelling of the affected limb developed 177 days after discontinuing linezolid administration. A computed tomography scan of the pelvic limb showed abscessation tracking along the caudal thigh, stifle and tibia, without evident communication with the stifle joint. The aerobic and anaerobic bacterial cultures of the exudate and fasciae tissue yielded no growth. The lameness and swelling resolved 14 days after treatment with surgical debridement and local vancomycin-impregnated pluronic gel administration, placement of a Jackson-Pratt drain and oral amoxicillin/clavulanic acid administration. At the time of this writing, the owner reported no recurrence of limb swelling, and the dog continued to be sound.

In another $\operatorname{dog}$ (No. 3 ), the draining tract recurred 52 days after discontinuing the linezolid therapy. Ultrasound examination of the local area showed a small bone fragment associated with the recurrent draining tract. The draining material was aspirated and submitted for aerobic culture and sensitivity tests. No organisms grew on the culture. The client declined surgical exploration, additional aerobic and anaerobic culture or amputation. The dog was euthanatized 80 days after discontinuing the linezolid therapy.

\section{Discussion}

Multimodal therapy of multidrug-resistant orthopaedic MRSP SSI with long-term oral linezolid was successful in two of the four dogs. The two dogs that received linezolid at previouslyreported dosages ${ }^{6}$ developed drug-associated side effects. All MRSP isolated in the present study were multidrug-resistant. Other medications demonstrated susceptibility based on the culture and sensitivity report, including trimethoprim-sulfa, amikacin, daptomycin, rifampin and vancomycin. Trimethoprim-sulfa or amikacin was given to all the dogs in the present report before considering linezolid administration, but 
trimethoprim-sulfa and amikacin therapy failed. The use of rifampin, vancomycin and linezolid has been reported in veterinary medicine for the treatment of MRSP infections. ${ }^{3,4}$ Linezolid was selected because it can be given orally in the long-term without the need for hospitalization and with a low risk of potential hepatic and renal side effects. By comparison, vancomycin can only be administered intravenously, and rifampin has a higher risk of hepatic or renal toxicity.

Treatment was unsuccessful in two patients. One of them developed abscessation of the previously affected limb approximately 6 months after discontinuing linezolid. Bacterial cultures from samples obtained before and during surgical debridement yielded no growth. The ability to detect bacteria in cultures may have been negatively impacted by the empirical antimicrobial therapy before sampling. Although the causative bacteria could not be determined, a recurrence of SSI was likely considering the proximity of the abscess to the previously infected surgical site. The other dog with recurrence had several avascular bone fragments removed for bacterial culture during surgical debridement. When the infection recurred, an additional small bone fragment was identified by ultrasound. This small bone fragment possibly was a continued nidus of infection. The dog also received the lowest linezolid concentration of all four patients. Although the trough serum drug level was twice the bacterial minimally inhibitory concentration (MIC), it is possible that the 24-hour area under curve time:MIC ratio or the local tissue concentration was inadequate for achieving bactericidal effect, leading to a treatment failure. ${ }^{7}$

Serum linezolid was tested in all dogs to ensure adequate therapeutic levels were achieved during therapy. Serum drug levels varied widely among the four dogs. The minimal serum concentrations measured in the present case series were higher than previously reported. ${ }^{6}$ Concurrent medication, dosage and formulation of linezolid, preexisting subclinical liver and kidney disease and differences in sample measurement methods could have contributed to the variability in serum drug concentration levels. The relatively wide range in serum levels observed in the present series would warrant further pharmacokinetic studies to help determine optimal linezolid dosage, dosing sequence, administration route and local tissue concentration.

The short-term adverse drug events observed following oral linezolid treatment in the presented case series included vomiting, nausea and anorexia. These events were not previously reported in veterinary patients but have frequently been reported in humans. ${ }^{4}$ Nausea or vomiting occurred in up to $23 \%$ of human patients receiving systemic linezolid. 8,9 The mechanism of gastrointestinal adverse events is not fully known. Alteration of the microbiome in the gastrointestinal tract has been proposed as a potential cause. ${ }^{9}$ Human patients receiving an injectable form of linezolid were less likely to experience nausea or vomiting compared with an oral form of linezolid in one report. ${ }^{9}$ Additionally, hospitalization with intravenous linezolid administration would have also allowed a more frequent drug level measurement and adjustment of drug dosage. We did not attempt to use injectable linezolid due to the need for long-term hospitalization, intravenous catheter placement, the increased risk of intravenous catheter-associated infection and cost. Nonetheless, hospitalization with intravenous linezolid may be considered in cases that developed severe gastrointestinal symptoms following oral linezolid.

The median duration of linezolid administration in the present report is longer than the 10 to 28 days recommended in human medicine. ${ }^{10}$ For the treatment of osteomyelitis and orthopaedic implant infections, a prolonged period of antimicrobial therapy is often necessary. In human studies, the median duration of linezolid for the treatment of orthopaedic implant infections ranged from 47 to 84 days, ${ }^{11,12}$ a duration similar to that of the present study. The dosage used in the current study ranged from 7 to $14 \mathrm{mg} / \mathrm{kg}$. The initial dosage was extrapolated from the previous veterinary reports. ${ }^{3,4}$ However, an adjustment to a lower dose was necessary in two of the four cases. A second trough level was performed to ensure adequate serum level was achieved. A more frequent serum drug level testing such as hospitalization with intravenous administration and daily serum level testing would have been beneficial, especially when handling a tertiary antimicrobial.

Regenerative anaemia was reported in one of the four dogs after receiving linezolid for 15 weeks. Anaemia was likely due to increased oxidative damage to erythrocytes. ${ }^{13}$ Anaemia and thrombocytopenia have been reported in $20 \%$ of human patients receiving long-term linezolid treatment, and routine haematological screening is recommended in human patients receiving long-term linezolid therapy. ${ }^{9}$ Deep pyoderma with $P$. aeruginosa infection occurred in one dog. This dermatological infection was likely multifactorial. The dog underwent hydrotherapy in an underwater treadmill. Although the incidence of pyoderma after aquatic therapy in dogs is not known, more than $30 \%$ of hydrotherapy pools used in human medicine are reportedly contaminated with $P$. aeruginosa. $^{14}$ In one report, $P$. aeruginosa was the most commonly identified bacteria that led to dorsal skin furunculosis after water immersion in dogs. ${ }^{15}$ Also, linezolid targets Gram-positive bacteria, which can disrupt the normal skin microbiome, subsequently increasing the risk of Gram-negative bacterial infection such as infections caused by Pseudomonas spp. Routine skin monitoring for infections of patients receiving linezolid is advised, especially in immune-compromised patients and patients with a history of Gram-negative bacterial skin infection.

Local vancomycin-impregnated pluronic gel was placed in two dogs at the time of surgical debridement. Local antimicrobial delivery implant is an adjunct therapy for the treatment of SSIs and provides a high concentration of an effective antimicrobial agent that would allow eradication of local bacteria. ${ }^{16}$ The cumulative elution concentration of vancomycin-impregnated pluronic gel is $6,336 \mu \mathrm{g} / \mathrm{mL}$ over 10 days, ${ }^{17}$ which can be toxic to osteoblasts and chondrocytes. ${ }^{18,19}$ Additional research to evaluate the associated risks and therapeutic benefits of antimicrobial impregnated pluronic gel is warranted.

This report has limitations. It was a retrospective study, the number of patients was small and the cases were heterogeneous. A larger case series would have been preferable for 
assessment of treatment efficacy but would be challenging since most SSI can be successfully treated with other antimicrobials without the need to administer linezolid.

This report provides insights for the clinical application, drug dosage and side effects of enteral linezolid therapy for the treatment of orthopaedic SSI. Although all dogs showed initial improvement of clinical signs, adverse drug events and treatment failure occurred in two out of four patients. As a tertiary antimicrobial, the use of linezolid for the treatment of SSI should be limited. If linezolid therapy is indicated by the culture and sensitivity report for the treatment of multidrug-resistant SSI after orthopaedic surgery, a multimodal approach is recommended that includes thorough debridement of necrotic tissue, removal of all infected implants and bacterial biofilm, careful extrapolation of doses based on serum trough levels and close monitoring of haematological parameters to prevent or minimize possible drug-related side effects and bacterial resistance.

\section{Authors' Contributions \\ P.-Y.C., D.J.M.-L., and R.A.H.-B., contributed to case man- agement and follow-up interview or examination. J.Y. contributed to data collection. P.-Y.C. and D.J.M.-L. con- tributed to manuscript preparation. R.H.-B. contributed to manuscript revision.}

\section{Funding}

None.

\section{Conflict of interest \\ None declared.}

\section{Acknowledgment}

The authors would like to acknowledge Dr. Amy Kapatkin's support and advice for manuscript preparation.

\section{References}

1 Roger C, Roberts JA, Muller L. Clinical pharmacokinetics and pharmacodynamics of oxazolidinones. Clin Pharmacokinet 2018;57(05):559-575

2 Bozdogan B, Appelbaum PC. Oxazolidinones: activity, mode of action, and mechanism of resistance. Int J Antimicrob Agents 2004;23(02):113-119

3 Foster JD, Trepanier LA, Ginn JA. Use of linezolid to treat MRSP bacteremia and discospondylitis in a dog. J Am Anim Hosp Assoc 2014;50(01):53-58
4 Papich MG, Papich MG. Selection of antibiotics for methicillinresistant Staphylococcus pseudintermedius: time to revisit some old drugs? Vet Dermatol 2012;23(04):352-360, e64

5 Razonable RR, Osmon DR, Steckelberg JM. Linezolid therapy for orthopedic infections. Mayo Clin Proc 2004;79(09):1137-1144

6 Slatter JG, Adams LA, Bush EC, et al. Pharmacokinetics, toxicokinetics, distribution, metabolism and excretion of linezolid in mouse, rat and dog. Xenobiotica 2002;32(10):907-924

7 Pea F, Furlanut M, Cojutti P, et al. Therapeutic drug monitoring of linezolid: a retrospective monocentric analysis. Antimicrob Agents Chemother 2010;54(11):4605-4610

8 Rubinstein E, Isturiz R, Standiford HC, et al. Worldwide assessment of linezolid's clinical safety and tolerability: comparatorcontrolled phase III studies. Antimicrob Agents Chemother 2003; 47(06):1824-1831

9 Bishop E, Melvani S, Howden BP, Charles PGP, Grayson ML. Good clinical outcomes but high rates of adverse reactions during linezolid therapy for serious infections: a proposed protocol for monitoring therapy in complex patients. Antimicrob Agents Chemother 2006;50(04):1599-1602

10 Pfizer. Zyvox (linezolid) prescribing information; 2015. Available from: http://labeling.pfizer.com/showlabeling.aspx?id=649. Accessed April 13, 2020

11 Legout L, Valette M, Dezeque H, et al. Tolerability of prolonged linezolid therapy in bone and joint infection: protective effect of rifampicin on the occurrence of anaemia? J Antimicrob Chemother 2010;65(10):2224-2230

12 Soriano A, Gómez J, Gómez L, et al. Efficacy and tolerability of prolonged linezolid therapy in the treatment of orthopedic implant infections. Eur J Clin Microbiol Infect Dis 2007;26(05):353-356

13 Wang T, Guo D, Dong X, Mu L. Effect of linezolid on hematological and oxidative parameters in rats. J Antibiot (Tokyo) 2014;67(06): 433-437

14 Moore JE, Heaney N, Millar BC, Crowe M, Elborn JS. Incidence of Pseudomonas aeruginosa in recreational and hydrotherapy pools. Commun Dis Public Health 2002;5(01):23-26

15 Cain CL, Mauldin EA. Clinical and histopathologic features of dorsally located furunculosis in dogs following water immersion or exposure to grooming products: 22 cases (2005-2013). J Am Vet Med Assoc 2015;246(05):522-529

16 Hayes G, Moens N, Gibson T. A review of local antibiotic implants and applications to veterinary orthopaedic surgery. Vet Comp Orthop Traumatol 2013;26(04):251-259

17 Thomas LA, Bizikova T, Minihan AC. In vitro elution and antibacterial activity of clindamycin, amikacin, and vancomycin from Rgel polymer. Vet Surg 2011;40(06):774-780

18 Shaw KA, Eichinger JK, Nadig N, Parada SA. In vitro effect of vancomycin on the viability of articular chondrocytes. J Orthop Trauma 2018;32(03):148-153

19 Edin ML, Miclau T, Lester GE, Lindsey RW, Dahners LE. Effect of cefazolin and vancomycin on osteoblasts in vitro. Clin Orthop Relat Res 1996;333:245-251 